\title{
APLICACIÓN DEL TEST DE FUKUDA PARA IDENTIFICACIÓN DE ALTERACIONES PROPIOCEPTIVAS EN PACIENTES PRE Y POSQUIRÚRGICOS DE EXTRACCIÓN DEL TERCER MOLAR, EN EL HOSPITAL "PABLO ARTURO SUÁREZ"
}

\author{
Susana Argüello P. ', Cristina Díaz C. ${ }^{2}$, Adrián Rubio ${ }^{3}$
}

\section{RESUMEN}

El presente estudio se realizó en los meses de julio-agosto de 2015, en el área de cirugía maxilofacial del hospital "Pablo Arturo Suárez", con el objetivo de determinar si existe una variación en cuanto a la propiocepción en pacientes que son extraídos los terceros molares, por medio de la aplicación del Test de Fukuda.

La investigación ejecutada fue de tipo exploratoria, prospectiva, observacional y longitudinal. La muestra obtenida fue de 30 pacientes entre 16 a 56 años, de los cuales 13 son hombres y 17 mujeres. Los criterios de inclusión fueron que tuviesen al menos dos terceros mo- lares uno a cada lado y que no presentasen patologías vestibulares, otitis, y alteraciones en la propiocepción por enfermedades del sistema nervioso. Se comprobó que existe una diferencia significativa $(p<0,013)$ en el desplazamiento en centímetros después de la extracción de los terceros molares, pero no existe una diferencia significativa en los grados de los giros.

Palabras clave: articulación temporomandibular, Propiocepción, Terceros molares, Test de Fukuda.

\section{ABSTRACT}

This study used the test Fukuda to

\footnotetext{
1 Pontificia Universidad Católica del Ecuador, Facultad de Enfermería, Quito, Ecuador (smarguello@puce.edu.ec).

2 Pontificia Universidad Católica del Ecuador, Facultad de Enfermería, Quito, Ecuador (acdiaz@puce.edu.ec).

${ }_{3}^{3}$ Pontificia Universidad Católica del Ecuador, Facultad de Enfermería, Quito, Ecuador (adrian_rb@hotmail.com).
} 
determine some variation in proprioception in patients whose third molars were removed in the area of maxillofacial surgery at Pablo Arturo Suarez' hospital conducted in the months of July-August 2015.

The research was exploratory, prospective, observational and longitudinal. The sample included 30 patients aged 16-56 years, 13 of whom were men and 17 women; the inclusion criteria was that they had two third molars on each side, which caused vestibular diseases, otitis, and proprioception alterations with nervous system diseases. This research found a significant difference ( $p$ $<0.013$ ) in the displacement in centimeters after removal of third molars, but there were no significant difference in the degrees of turns.

Keyword: temporomandibular joint, proprioception, third molars, Fukuda test.

\section{INTRODUCCIÓN}

La propiocepción hace referencia a la capacidad del cuerpo de detectar el movimiento y posición de las articulaciones. Es importante en los movimientos comunes que realizamos diariamente $y$, en especial, en los movimientos deportivos que requieren una coordinación especial.

Los trastornos temporomandibulares no solo se pueden asociar debido a su cercanía con la posición de la mandíbula y del cráneo, sino también con la columna cervical, torácica, lumbar, la musculatura supra e infrahioidea, los hombros que funcionan como una unidad biomecánica. Por lo tanto, los cambios en cualquiera de estos componentes podrían desencadenar alteraciones en el sistema estomatognático (Montero y Denis, 2013).
Una alteración a nivel de la articulación temporomandibular (ATM) puede aparecer a cualquier edad y en cualquier circunstancia de la vida, ya que las posibilidades son diversas y van desde un simple golpe en la zona, hasta el aparecimiento de nuevas estructuras óseas (tercer molar).

Son muchos los pacientes que sufren de inestabilidad, mareos, cefaleas, jaquecas, vértigo, dolores de columna cervical, lumbalgias o ciáticas repetitivas, entre otras patologías, que acuden a consulta a la unidad de cirugía maxilofacial del hospital "Pablo Arturo Suárez", y que debido a la falta de coordinación y conocimiento no se remite al área de rehabilitación física del hospital "Pablo Arturo Suárez" para efectuar un trabajo interdisciplinario. 
El test de Fukuda es un estudio de equilibrio dinámico que evalúa la descompensación lateral e hipertonía de un individuo, en el que se pide al paciente que con los ojos cerrados y los brazos extendidos a $90^{\circ}$ hacia al frente realice una marcha modo soldado (en su propio terreno) de 50 pasos alrededor de un minuto; si la persona se mantiene en el mismo sitio, la prueba es negativa y si existen giros mayores a $30^{\circ} \mathrm{o}$ el paciente avanza más de dos metros al cabo de los 50 pasos, es positiva. El lado de la desviación se le considerará hipofuncional (Bri$\cot B, 2008)$.
A pesar de algunas evidencias en contra, la mayoría de las investigaciones demuestran que los disturbios musculares posturales son más frecuentes en pacientes con trastornos temporomandibulares y establecen algún tipo de correlación entre el tipo de oclusión, la existencia de signos y síntomas de los trastornos temporomandibulares, dimensiones craneofaciales y el desequilibrio postural (Montero 2012); por lo cual se realiza esta investigación para determinar por medio de la aplicación del test de Fukuda la variación de la propiocepción previa y posterior a la extracción del tercer molar en los pacientes del área de cirugía maxilofacial del hospital "Pablo Arturo Suárez".

\section{HIPÓTESIS}

Existe una mejoría en la propiocepción de pacientes de la unidad de cirugía maxilofacial del hospital "Pablo Arturo
Suárez" luego de la extracción de los terceros molares.

\section{OBJETIVOS}

Identificar, por medio de la aplicación del test de Fukuda, la variación de la propiocepción, posterior a extracción del tercer molar en pacientes del área de cirugía maxilofacial del hospital "Pablo Arturo Suárez".

\section{MATERIALES Y MÉTODOS}

Es un estudio tipo exploratorio, prospectivo, observacional, longitudinal. El grupo de estudio son los pacientes que han sido sometidos a extracción de los terceros molares, quienes fueron evaluados luego de siete días de la extracción.
El estudio fue realizado en 30 pacientes entre las edades de 16 y los 56 años, que tuvieron al menos dos terceros molares uno a cada lado, que no presentaban alteraciones en la propiocepción como consecuencia de alguna patología 
base como síndrome de Menier, Otitis, inicios de Parkinson, además de patologías asociadas a problemas vestibulares.

Para el género femenino, el promedio de edad es 23.7 años ( $\mathrm{DE}=9.4$ años), con un valor mínimo de 16 y un máximo de 56. Para el género masculino, el promedio de edad es 24.22 años ( $D E=11.51$ años), con un valor mínimo de 16 y un máximo de 51.

Para realizar el test de Fukuda el paciente se ubicaba en un sitio con el menor ruido posible, descalzo, con los ojos cerrados, marcha con una altura del muslo a $45^{\circ}$, la cabeza en posición neutra, los maxilares en posición de reposo (sin con- tacto dental), los brazos a 90 grados de flexión. Se filmó la ejecución de la prueba.

Las variables estudiadas fueron las siguientes: diferencia del desplazamiento en cm del test de Fukuda pre- y posextracción de los terceros molares y variación de los grados de giro del test de Fukuda pre-y poscirugía.

El Análisis estadístico se lo realizó sobre la base de medidas de tendencia central, de dispersión, y prueba " $T$ " de comparación de medias relacionadas, usando las herramientas informáticas de Microsoft Excel versión 2010 y SPSS versión 23.

\section{RESULTADOS}

Tabla 1. Medidas de tendencia central y dispersión de los parámetros del Test de Fukuda

\begin{tabular}{|l|c|c|c|c|}
\hline Media & $\begin{array}{c}\text { Desplazamiento } \\
\text { preextracción } \\
(\mathrm{cm})\end{array}$ & $\begin{array}{c}\text { Desplazamiento } \\
\text { posextracción } \\
(\mathrm{cm})\end{array}$ & $\begin{array}{c}\text { Grados de } \\
\text { desviación } \\
\text { preextracción }\end{array}$ & $\begin{array}{c}\text { Grados dé } \\
\text { desviación } \\
\text { posextracción }\end{array}$ \\
$\begin{array}{l}\text { Desviación estándar } \\
\text { Mínimo }\end{array}$ & 39,3 & 31,3 & 55,6 & 52,5 \\
Máximo & 10 & 17,9 & 38,3 & 37,8 \\
\hline
\end{tabular}

En la tabla 1 se observa que existe disminución del desplazamiento en la aplicación del test de Fukuda, después de la extracción de los terceros molares. La media de desplazamiento precirugía es de $39.33 \mathrm{~cm}$ y poscirugía es de 31.3 $\mathrm{cm}$. Existe una diferencia entre datos máximos pos y pre de $20 \mathrm{~cm}$. El dato máximo de distancia recorrida antes de la extracción es de $110 \mathrm{~cm}$, y después de la cirugía el dato máximo es de $90 \mathrm{~cm}$. 
Otro parámetro del test de Fukuda son los grados de desviación, los cuales en la tabla 1 no presentan un gran cambio antes y después de la cirugía de los terceros molares. La media posextracción es de $52.5^{\circ}$ y preextracción es de
55.6 . La diferencia entre datos máximos pos y pre de $5^{\circ}$. El dato máximo de grados de desviación antes de la extracción es de $130^{\circ}$, y después de la cirugía el dato máximo es de $125^{\circ}$.

Tabla 2. Prueba de muestras relacionadas de la distancia de desplazamiento pre- y posextracción del tercer molar

\begin{tabular}{|l|c|c|c|c|c|}
\hline & \multicolumn{2}{|c|}{$\begin{array}{c}\text { Diferencias relacionadas } \\
95 \% \text { Intervalo de confianza } \\
\text { para la diferencia }\end{array}$} & Media & Desv. est. & Sig. (bilateral). \\
\cline { 2 - 5 } & $\begin{array}{l}\text { Inferior } \\
\text { Superior }\end{array}$ & $-14,2$ & -8 & 16,5 & 0,013 \\
\hline $\begin{array}{l}\text { Diferencia entre } \\
\text { distancia posextracción y } \\
\text { distancia preextracción. }\end{array}$ & $-1,8$ & -8 & & \\
\hline
\end{tabular}

En la tabla 2 de la prueba estadística de diferencias relacionadas, entre la distancia posextracción y preextracción de los terceros molares existe un $\mathrm{p}<0,05$ (0.013), concordando con la tabla 1, que se observaba una disminución de la distancia poscirugía. Existe una media de $8 \mathrm{~cm}$ de la diferencia entre la distancia pos- y preextracción de los terceros molares. El menor dato de la diferencia entre distancia pos- y preextracción de los terceros molares es de -1.8 y el máximo dato es de $-14,2$.

Tabla 3. Prueba de muestras relacionadas de los grados de desviación pre- y posextracción del tercer molar

\begin{tabular}{|c|c|c|c|c|c|}
\hline & \multicolumn{2}{|c|}{$\begin{array}{l}\text { Diferencias relacionadas } \\
95 \% \text { Intervalo de confianza } \\
\text { para la diferencia }\end{array}$} & \multirow[t]{2}{*}{ Media } & \multirow[t]{2}{*}{ Desv. est. } & \multirow[t]{2}{*}{ Sig. (bilateral). } \\
\hline & Inferior & Superior & & & \\
\hline $\begin{array}{l}\text { Diferencia entre grados de } \\
\text { desviación posextracción } \\
\text { y preextracción. }\end{array}$ & $-9,8$ & $-16,2$ & $-3,2$ & 34,8 & 0,62 \\
\hline
\end{tabular}


En la tabla 3 de la prueba estadística de diferencias relacionadas, entre los grados de desviación posextracción y preextracción de los terceros molares existe un $\mathrm{p}>0,05$ (0.622), concordando con la tabla 1, que se observaba que no existe una disminución considerable de los grados de desviación poscirugía.
Existe una media de $-3.2^{\circ}$ de la diferencia entre los grados de desviación post- y preextracción de los terceros molares. El menor dato de la diferencia entre los grados de desviación pos- y preextracción de los terceros molares es de $-9.8^{\circ}$ y el máximo dato es de $-16,2^{\circ}$.

\section{DISCUSIÓN}

El mantenimiento de la postura está dado por el sistema nervioso central y periférico, el tono muscular, la propiocepción, el sistema vestibular, el sistema visual, la alineación estructural. Si algunos de estos elementos se encuentran alterados la postura se modifica (Toussaint, Y. 2008).

El test de Fukuda evalúa la propiocepción en la postura, y si existe una disfunción periférica, el paciente se desvía más de 30 grados desde el eje sagital. Además, los valores de vaivén lateral amplios y permanentes sugieren una patología central (Schneider D, 2011).

El mencionado test se realiza con el cierre de los ojos; pues sin visión, es casi imposible pisar en el mismo lugar y no desviarse $30^{\circ}$. Estudios del test de Fukuda con estímulos visuales del uso de gafas de realidad virtual corroboran que no existen modificación en los resultados del test, pero la influencia vestibular con pruebas calóricas de irrigación en el oído sí modifican los resultados del test de Fukuda aumentando los grados de desviación hacia el oído estimulado (Marques B, 2014).

Se han realizado estudios modificando el test para determinar si los factores de ritmo impuesto, de doble tarea, o con el cuello doblado influyen en los grados de desviación, y se ha observado que con la cervical flexionada se aumentaron los grados de desviación (Toussaint, Y. 2008).

La aplicación del test de Fukuda con verificación craneocorpografía digital, en pacientes con síndromes cervicales agudos, presenta variación en los grados de desviación (Alpini, D. 2013).

Recientemente se han relacionado alteraciones oclusales funcionales con enfermedades posturales, en su mayoría sobre una base clínica. Sin embargo, algunos aspectos anatómicos y fisiológicos son todavía desconocidos. Sujetos 
con una mala oclusión de clase II al ser evaluados en plataformas de equilibrio, presentan una postura con alteración en la cadena muscular anterior, mientras que los sujetos con una mala oclusión clase III presentan una postura con alteración en la cadena muscular posterior (Nobili A, Adversi R. 2006).

El uso prolongado de aparatos bucales tipo MORA induce a algunas fluctuaciones en la actitud postural dinámica al ser evaluados con el Test Fukuda. Los efectos de retroalimentación son graduales después de retirar la férula mandibular (Milani, RS. 2011).
En este estudio se observa en la tabla 1 y 3 que existe una variación significativa en el test de Fukuda después de extraer el tercer molar en el parámetro de desplazamiento pero no en la desviación en grados. Sin embargo, las limitaciones que tiene esta investigación se deben al tiempo de ejecutada la segunda prueba de Fukuda, pues el paciente todavía estaba en el proceso de desinflamación propia de la intervención quirúrgica, por lo que se hacen necesarias evaluaciones luego de un tiempo más prolongado para evaluar la variación de la propiocepción en el tiempo.

\section{CONCLUSIONES}

Esta investigación demuestra que la mala posición de los terceros molares puede alterar la propiocepción, que en última instancia es necesaria para mantener un adecuado control postural, por lo cual se precisa la extracción de estos molares, no solo con el objetivo de mejorar estéticamente la mordida, sino también para que la información propioceptiva que envía la ATM al sistema nervioso central pueda ser la correcta, para que este elabore las respuestas motoras adecuadas que determinan un adecuado equilibrio corporal.

A la extracción de los terceros molares existe una disminución de las alteraciones propioceptivas, específicamente en el desplazamiento longitudinal, mientras que las desviaciones en grados son poco significativas. 


\section{BIBLIOGRAFÍA}

Alpini D. (2004). Evaluation of head-to-trunk control in whiplash patients using digital CranioCorpoGraphy during a stepping test. Gait and Posture. 22 (4) 308-316. Recuperado de: http://www. gaitposture.com/article/S09666362(04)00202-4/abstract.

Bricot B. (2008). Postura normal y postura patológica. Revista IPP, 1 (2). 1-13.

Marques B. (2010). Influencia de la estimulación vestibular y visual en fracción de banda para caminar. Springer link. 183 (4) 457 -463. Recuperado de: http://link.springer.com/article/10.100 7\%2Fs00221-007-1063-4.

Milani RS. De Perière D. Lapeyre L. Pourreyron L. (2000). Relationship between dental occlusion and posture. Cranio. 18(2)34 - 127. Recuperado de: http:// www.ncbi.nlm.nih.gov/pubmed/1120 2823.

Montero J. Denis. (2013). Los trastornos temporomandibulares y la oclusión dentaria a la luz de la posturología moderna. 50 (2). Recuperado de: http://scielo.sld.cu /scielo.php?pid=S0034750720130004 00008\&script=sci_arttext\&tlng=pt.
Nobili A, Adversi R. (2006). Relationship between posture and Occlusion: a clinical and experimental investigation. Cranio 14(4):85 - 274. Recuperado de: http:// www.ncbi.n/m.nih.gov/pubmed/9110 621.

Schneider D (2011). Cranio-corpo-graphy. A neurootological screening test. Acta Otorhinolaryngol Belg. 45(4). Recuperado de: http://www.ncbi.nlm.nih.gov /pubmed/1767670.

Toussaint Y. (2008). What are the factors responsible for the deviation in stepping on the spot? 435 (1) 60 - 64. Recuperado de: http://www.sciencedirect.com/sci ence/article/pii/S030439400800181X. 